\title{
Formation of Perfluoroalkyl Fullerene Alkylphosphonic Acid Self-Assembled Monolayers on Aluminum Oxide
}

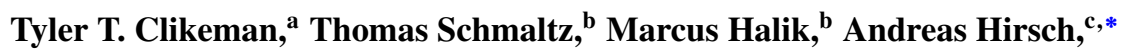 \\ Steven H. Strauss, ${ }^{\text {a,* }}$ and Olga V. Boltalina ${ }^{a, *, z}$ \\ ${ }^{a}$ Department of Chemistry, Colorado State University, Fort Collins, 80523, USA \\ ${ }^{b}$ Organic Materials \& Devices (OMD), Institute of Polymer Materials, University Erlangen-Nürnberg, \\ 91058 Erlangen, Germany \\ ${ }^{c}$ Department of Chemistry and Pharmacy, University Erlangen-Nürnberg, 91054 Erlangen, Germany
}

The possibility of fabricating self-assembled monolayers (SAMs) from alkylphosphonic acids functionalized with trifluoromethyl derivatives of $\mathrm{C}_{60}$ on a metal oxide surface has been demonstrated in this work. Using several trifluoromethylfullerenes (TMFs) of different compositions and isomeric structures, we explored their relative reactivities in the Bingel-Hirsch cycloaddition reactions with 11-(diethoxyphosphoryl)undecyl methyl malonate for the first time. Only two isomers of $\mathrm{C}_{60}\left(\mathrm{CF}_{3}\right)_{10}$ showed fairly selective monoadduct formation, whereas compounds with lower $\mathrm{CF}_{3}$ content showed no selectivity. Converting the phosphonic ester into a phosphonic acid moiety enabled the successful formation of self-assembled monolayers of the TMF-alkylphosphonic acid on aluminum oxide surfaces. Comparison with analogous $\mathrm{SAMs}$ made with underivatized $\mathrm{C}_{60}$ showed that TMFs form more hydrophobic surfaces.

(C) The Author(s) 2017. Published by ECS. This is an open access article distributed under the terms of the Creative Commons Attribution 4.0 License (CC BY, http://creativecommons.org/licenses/by/4.0/), which permits unrestricted reuse of the work in any medium, provided the original work is properly cited. [DOI: 10.1149/2.0281706jss] All rights reserved.

(cc) BY

Manuscript submitted December 13, 2016; revised manuscript received February 23, 2017. Published March 7, 2017. This paper is part of the JSS Focus Issue on Nanocarbons - In Memory of Sir Harry Kroto.

Considerable progress has been made in the past decade on the synthesis, purification and fundamental properties of perfluoroalkyfullerenes (PFAFs); their potential applications span the fields of organic electronics, imaging of biological objects, and energy conversion devices. ${ }^{1}$ Incorporation of PFAFs in organic optoelectronic devices may be carried out using solution processing, or vapor deposition, enabled by improved solubility and thermal stability compared to commonly used organofullerenes. In this work, the formation of self-assembled monolayers (SAMs) of PFAF functionalized alkylphosphonic acids on aluminum oxide surfaces has been probed for the first time.

Use of SAMs for modification of electrical properties of various inorganic substrates has been proven as an effective method that may lead to improved conductivity and overall performance of organic optoelectronic devices. ${ }^{2}$ For example, one-dimensional metal oxide $\left(\mathrm{MO}_{x}\right)$ nanostructures show promise as new components in high-tech electronics. ${ }^{3}$ However, $\mathrm{MO}_{x}$ nanostructures suffer from substandard charge transfer across the particle/particle interfaces, so mainstream application has yet to be achieved. Tunable electron acceptors have been attached to $\mathrm{ZnO}$ nanorods in the form of self-assembled monolayers (SAMs) in order to tune the electrical properties of the $\mathrm{ZnO}$ nanostructures. ${ }^{4-6}$ The Halik group has shown that a SAM with $\mathrm{C}_{60^{-}}$ acceptor units can accept and accumulate electronic charges when incorporated into organic thin-film memory transistors. ${ }^{5} \mathrm{The}_{60} \mathrm{C}_{60}$ derivatives that differed by alkyl linkers of different chain lengths were synthesized and formed SAMs on the surface of $\mathrm{ZnO}$ nanorods with different insulating properties to tune the electrical properties of $\mathrm{ZnO}$ nanorods. ${ }^{6}$ The electron withdrawing $\mathrm{C}_{60}$ moiety was able to electronically couple with $\mathrm{ZnO}$ and as a result, the electrical performance of $\mathrm{ZnO}$ nanorods was improved. Further performance optimizations on SAM modified $\mathrm{ZnO}$ nanorods, and other $\mathrm{MO}_{x}$ such as $\mathrm{AlO}_{x}$, require more efficient electron transfer and longer lifetimes of the reduced $\mathrm{C}_{60}$ moiety. Attractive candidate materials that may fulfill these prerequisites are PFAFs, which not only exhibit superior electron acceptor properties, but also possess chemical and thermal robustness. SAMs consisting of PFAFs could ease the transfer of electrons and increase the lifetimes of the charged state (Figure 1). A variety of PFAFs can

*Electrochemical Society Member.

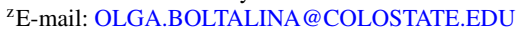

be tested to discover the optimal electron-withdrawing strength and degree of fluorine insulation.

In this work, we have explored Bingel-Hirsch cycloaddition reactions with several trifluoromethylfullerenes (TMFs) $\mathrm{C}_{60}\left(\mathrm{CF}_{3}\right)_{n}$ that differ by composition ( $\mathrm{n}=2,4$, and 10) and by the isomeric structures in the case of $\mathrm{C}_{60}\left(\mathrm{CF}_{3}\right)_{10}$. The Schlegel diagrams of the TMFs used in this study are shown in Figure 2. Formation of SAMs on $\mathrm{AlO}_{\mathrm{x}}$ surface was studied using one of the TMFs that demonstrated higher selectivity.

\section{Experimental}

Synthesis of 11-(tetrahydropyranyloxy)-1-bromoundecane.PPTS (0.230 g, $0.907 \mathrm{mmol})$ and DHP $(0.52 \mathrm{~mL}, 5.7 \mathrm{mmol})$ were

\subsection{1}

0.26

$0.57 \mathrm{~V}$

$\mathrm{C}_{60}\left(\mathrm{CF}_{3}\right)_{n}$ reduction potentials versus $\mathrm{C}_{60}$

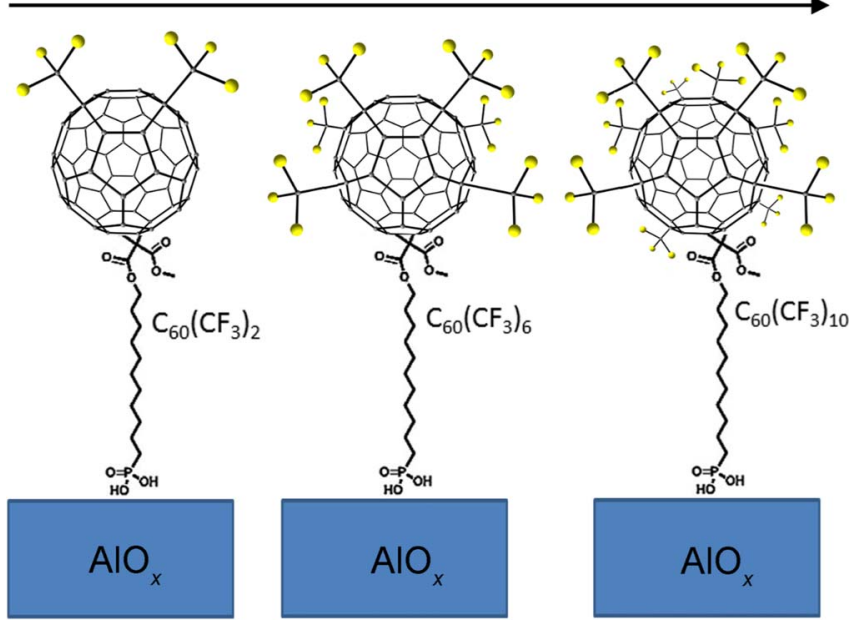

Figure 1. Schematic representation of aluminum oxide surfaces coated with SAMs using different PFAFs. Reduction potentials of PFAFs without phosphonic acid linkages are shown. 

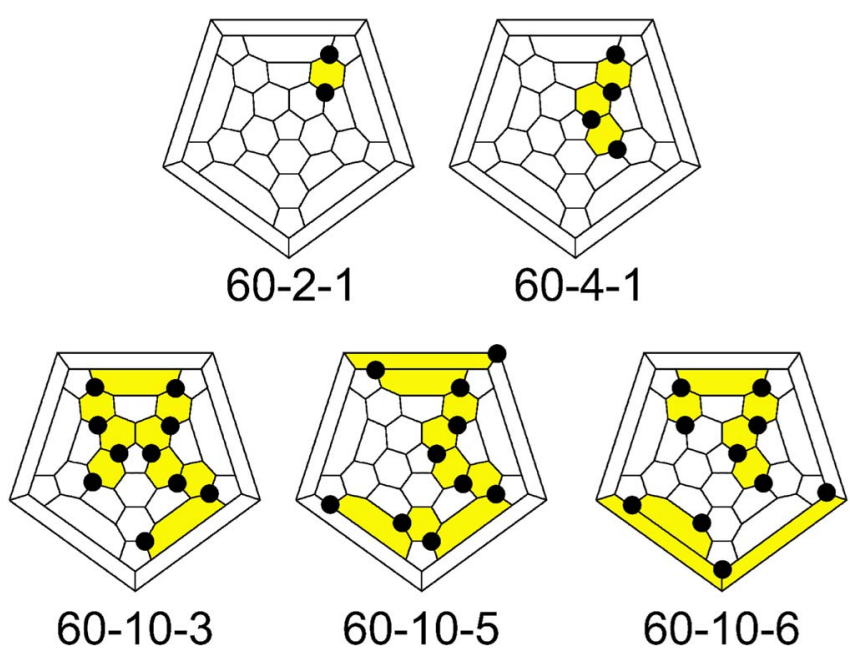

Figure 2. Schlegel diagrams of trifluoromethylfullerenes used in this work. Solid black dots denote the location of $\mathrm{CF}_{3}$ groups on the carbon cage; The hexagons occupied with $\mathrm{CF}_{3}$ groups are colored in yellow to visually assist in distinguishing addition patterns on different isomers. In TMF notations, the first number designates number of carbon atoms on the cage, the second number designates the number of $\mathrm{CF}_{3}$ groups, and the third number designates the number of the isomer.

added to 11-bromo-1-undecenol (1.14 g, $4.54 \mathrm{mmol})$ in DCM (100 $\mathrm{mL})$. After $16 \mathrm{~h}$ the reaction mixture was washed with a saturated $\mathrm{NaHCO}_{3}$ solution $(300 \mathrm{~mL})$ and separated. The aqueous layer was washed with additional DCM $(100 \mathrm{~mL})$. The two organic solutions were combined and washed with a saturated $\mathrm{NaCl}$ solution $(200 \mathrm{~mL})$ then dried over $\mathrm{Na}_{2} \mathrm{SO}_{4}$. The solvent was removed by rotovap and the product was obtained as a colorless oily liquid. Yield: $1.55 \mathrm{~g}, 100 \%$.

Synthesis of diethyl [18-(tetrahydropyranyloxy)undecyl] phosphonate.-A yellow mixture of 11-(tetrahydropyranyloxy)-1bromoundecane $(1.07 \mathrm{~g}, 3.19 \mathrm{mmol})$ and triethylphosphite $(5.46 \mathrm{~mL}$, $31.9 \mathrm{mmol}, 10$ equiv.) was refluxed for 6 hours (oil bath temperature $=170^{\circ} \mathrm{C}$ ). The excess triethylphosphite was removed by vacuum at $165^{\circ} \mathrm{C}$ and a yellow-orange, oily liquid remained. The crude product was used in the next step without further purification and isolation.

Synthesis of 11-(diethoxyphosphoryl)-1-undecanol.-A mixture of diethyl [18-(tetrahydropyranyloxy)undecyl] phosphonate (assume $3.19 \mathrm{mmol})$, ethanol (140 mL), and PPTS $(86.1 \mathrm{mg}, 0.343 \mathrm{mmol})$ were refluxed for 3 hours (oil bath temperature $=100^{\circ} \mathrm{C}$ ). The solvent was removed by rotovap and a yellow, oily liquid remained. The reaction mixture still contained unreacted PPTS which was removed on a small silica gel column. The product was purified by flash-chromatography on silica gel with ethyl acetate. Yield $0.435 \mathrm{~g}, 1.41 \mathrm{mmol}, 44 \%$ yield from 11-(tetrahydropyranyloxy)-1-bromoundecane.

Synthesis of 11-(diethoxyphosphoryl)undecyl methyl malonate.-A solution of methylmalonyl chloride $(0.23 \mathrm{~mL}$ in $30 \mathrm{~mL}$ DCM, $2.11 \mathrm{mmol}, 1.5$ equiv.) was added dropwise over the course of $30 \mathrm{~min}$. to a solution of 11-(diethoxyphosphoryl)-1undecanol $(0.435 \mathrm{~g}, 1.41 \mathrm{mmol})$ and $\mathrm{Et}_{3} \mathrm{~N}(0.30 \mathrm{~mL}, 2.11 \mathrm{mmol}, 1.5$ equiv.) in DCM $(80 \mathrm{~mL})$ at $0^{\circ} \mathrm{C}$. The solution was stirred for $2 \mathrm{~h}$ at $0^{\circ} \mathrm{C}$ then for $20 \mathrm{~h}$ at room temperature. The solvent was removed by rotovap and a yellow oil remained. The oil was dissolved in ethyl acetate and purified by flash-chromatography on silica with ethyl acetate and methanol. The product was collected as a colorless, oily liquid. Yield: $0.147 \mathrm{~g}, 0.375 \mathrm{mmol}, 25.5 \%$.

Synthesis of (60-10-3)(phosphonic ester)._-Under nitrogen and exclusion of light, 60-10-3 $(35.7 \mathrm{mg}, 25.3 \mathrm{mmol})$ was dissolved in degassed toluene $(18 \mathrm{~mL})$ at room temperature. Subequently, 11(diethoxyphosphoryl)undecyl methyl malonate $(0.20 \mathrm{~mL}, 0.60$ equiv.) and $\mathrm{I}_{2}(1.80,0.76$ equiv.) were added. A solution of DBU in toluene ( 0.79 equiv.) was added dropwise over the course of seven minutes. The reaction was monitored by TLC and was stopped after $20 \mathrm{~h}$ when unreacted 60-10-3 was no longer observed. White precipitate was filtered from a dark brown solution and the solution was subjected to flash chromatography on silica with toluene and toluene/methanol mixtures. The fractions containing (60-103)(phosphonic ester) monoadducts were further purified by HPLC in toluene. The product was analyzed by ${ }^{1} \mathrm{H},{ }^{19} \mathrm{~F}$, and ${ }^{31} \mathrm{P}$ NMR and MALDI mass spectrometry. Two peaks were present in the mass spectrum; $1830 \mathrm{~m} / \mathrm{z}$ is 14 more than desired product and unknown, 1748 $\mathrm{m} / \mathrm{z}$ is $\mathrm{C}_{60}\left(\mathrm{CF}_{3}\right)_{9}$ (malonate/phosphonic ester) +1 , which corresponds to the loss of one $\mathrm{CF}_{3}$ group in the mass spec experiment. Yield: $14.7 \mathrm{mg}, 32 \%$ yield based on starting $\mathbf{6 0 - 1 0 - 3}$.

Synthesis of (60-10-3)(phosphonic acid).-A dry dichloromethane solution $(5 \mathrm{~mL})$ of (60-10-3)(phosphonic ester) $(11.7 \mathrm{mg}$, $6.44 \mathrm{mmol})$ was cooled in an ice bath and $\mathrm{BrSiMe}_{3}(0.1 \mathrm{~mL}, 38$ mmol, 5.9 equiv.) was slowly added. The ice bath was removed after 2 hours and the soluiton was stirred for an additional 19 hours. Toluene, hexamethyldisiloxane and the excess of bromotrimethysilane were removed under vacuum. To remove any remaining traces of reagents, dry toluene $(5.0 \mathrm{~mL})$ was added and subsequently removed under vacuum. The residue was dissolved in $\mathrm{CHCl}_{3}(5 \mathrm{~mL})$ and then $\mathrm{MeOH}(5 \mathrm{~mL})$ and $\mathrm{H}_{2} \mathrm{O}(1 \mathrm{~mL})$ were added at room temperature and stirred for 2 hours. The solvents, except for $\mathrm{H}_{2} \mathrm{O}$, were removed under vacuum. Acetone was added and the acetone $/ \mathrm{H}_{2} \mathrm{O}$ azeotrope was removed under vacuum. The solid was dissolved in $\mathrm{CHCl}_{3}$, dried over $\mathrm{Na}_{2} \mathrm{SO}_{4}$, and then filtered. This entire reaction scheme was repeated several times. In some cases, $\mathrm{HCl}_{(a q)}$ was substituted for $\mathrm{H}_{2} \mathrm{O}$ or mixtures of $\mathrm{CHCl}_{3} / \mathrm{MeOH}$ were used for final collection. The product was analyzed by ${ }^{1} \mathrm{H},{ }^{19} \mathrm{~F}$, and ${ }^{31} \mathrm{P}$ NMR and MALDI mass spectrometry. The peaks in NMR have the same chemical shifts as starting (60-10-3)(phosphonic ester), but are more broadened. Proton peaks corresponding to the ester moiety were reduced. MALDI mass spectra indicated that there was a mixture of partially converted products, unreacted (60-10-3)(phosphonic ester), and (6010-3)(phosphonic acid). This mixture was used for SAM formation, since only the completely converted products form strong bonds and the esters and half esters can be washed away from the strongly bound SAM.

SAM characterization techniques.-X-Ray reflectivity Specular X-ray reflectivity (XRR) measurements and Grazing Incidence X-ray Diffraction (GIXD) experiments were carried out at beamline ID10 at the European Synchrotron Radiation Facility in Grenoble using $22 \mathrm{keV} \mathrm{X-rays,} \mathrm{the} \mathrm{details} \mathrm{of} \mathrm{the} \mathrm{measurements} \mathrm{were} \mathrm{described}$ elsewhere. 15

Preliminary FET testing.-FET devices using (60-10-3)(PA)SAMs were tested (Figure SI-4). Several different transistor setups and layouts (top contact, bottom contact, patterned by photolithography/ E-beam lithography, and shadow masks) were fabricated, but in all cases the drain current across the transistor channel did not exceed the leakage current through the SAM. In case of the shadow mask patterned devices during the gold evaporation for the source/drain electrodes, some gold leached into the transistor channel leading to an ohmic part of the current-voltage curve. Due to this leach, the drain current was lifted above the leakage current level and a current modulation with the gate voltage could be observed. This demonstrates that, in general, the devices worked. However, the charge transport in the SAM layer was so poor that it was hidden behind the leakage current in all other devices. A charge carrier mobility of approximately $10^{-7} \mathrm{~cm}^{2} /(\mathrm{Vs})$ (approximately two orders of magnitude lower than in the non-fluorinated $\mathrm{C}_{60}-\mathrm{SAMs}$ ) could be estimated. However, the devices with gold in the channel cannot really be considered FETs and none of the other devices worked. Various experiments to increase the 
thickness of the dielectric were attempted in order to reduce the leakage current, but did not improve device performance.

\section{Results and Discussion}

A limited number of reports on chemical derivatization of PFAFs involved Bingel-Hirsch and Diels-Alder cycloadditions to $\mathrm{C}_{70}\left(\mathrm{CF}_{3}\right)_{n}$ $(\mathrm{n}=8 \text { and } 10)^{7-9}$ and nucleophilic additions of $\mathrm{CN}^{-}$to TMFs of $\mathrm{C}_{60}$ and $\mathrm{C}_{70}$ with varying numbers of trifluoromethyl groups. ${ }^{10-12}$ Previously, selection of $\mathrm{C}_{70}\left(\mathrm{CF}_{3}\right)_{n}(\mathrm{n}=8$ and 10) for cycloaddition chemistry was based on the hypothesis that the presence of a reactive double bond on the $\mathrm{C}_{70}$ cage might promote regiospecific adduct formation, as proven to occur for both $\mathrm{C}_{70}$ TMFs. However, the $\mathrm{C}_{70}\left(\mathrm{CF}_{3}\right)_{n}$ $(\mathrm{n}=8$ and 10) compounds do not have sufficiently different electronic properties from those of parent fullerene, and thus they were excluded from the current study.

In order to covalently link a TMF electron acceptor to the $\mathrm{AlO}_{\mathrm{x}}$ surface, a phosphonic acid moiety has to be attached to a fullerene core. No experimental or theoretical work has been done on the BingelHirsch cycloaddition chemistry with $\mathrm{C}_{60}\left(\mathrm{CF}_{3}\right)_{n}$ compounds, prior to this study, despite the fact that many $\mathrm{C}_{60}$ TMFs can be prepared with reasonable yields and purity. The selected compounds (shown on Figure 2) have a wide range of reduction potentials that allow electronic tuning probing to be tested effectively.

The synthesis of 11-(diethoxyphosphoryl)undecyl methyl malonate and subsequent Bingel-Hirsch reactions were carried out according to modified literature procedures (Scheme 1). ${ }^{4-6}$

We found that selectivity of a Bingel-Hirsch reaction with phosphonic acid malonatic ester greatly depended on the TMF substrate. Furthermore, the number of phosphonic ester (PE) additions and isomers formed varied significantly, depending on the TMF substrate. In general, the derivatives with more $\mathrm{CF}_{3}$ groups, i.e., $\mathrm{C}_{60}\left(\mathrm{CF}_{3}\right)_{10}$ isomers, were more selective than derivatives with two or four $\mathrm{CF}_{3}$ groups. In contrast, reactions with 60-2-1 and 60-4-1 resulted in far too many products that were inseparable by combinations of flash chromatography and HPLC. These product mixtures exhibited complex and broad ${ }^{19} \mathrm{~F}$ NMR signals which indicated the formation of many products (Figures SI-1, SI-2). Furthermore, significant amounts of insoluble products were formed during the reactions, which is not common for a typical Bingel-Hirsch reaction. Further work with 60-2-1 and 60-4-1 adducts toward SAMs was therefore not pursued.

On the other hand, Bingel-Hirsch reactions with 60-10-3, 60-10-5, and 60-10-6 were more selective and did not produce as many products and undesirable insoluble materials. It appears that the presence of many $\mathrm{CF}_{3}$ groups on the fullerene core helps direct the malonate additions to the unique sterically unhindered unsubstituted cage regions present in $\mathrm{C}_{60}\left(\mathrm{CF}_{3}\right)_{10}$ isomers, which was not the case for the less substituted TMFs 60-2-1 and 60-4-1. Monoadducts were separated from bis- and trisadducts by flash chromatography with toluene/methanol mixtures and then the major monoadducts were further purified with HPLC in toluene.

The best result was obtained in the reaction with 60-10-3: a single, predominant isomer of (60-10-3)(PE) (PE is phosphonic ester) was formed and purified by HPLC in toluene using a Buckyprep column; the isolated yield was $32 \%$ based on starting 60-10-3. The ${ }^{19} \mathrm{~F}$ NMR spectrum of this product exhibited a ten-multiplet spectrum with similar chemical shifts to parent $\mathbf{6 0 - 1 0 - 3}$, indicating that the addition pattern of the ten $\mathrm{CF}_{3}$ groups remained unchanged upon phosphonic ester addition (Figure 3). A peak at $1748 \mathrm{~m} / \mathrm{z}$ in the MALDI mass spectrum corresponded to $\mathrm{C}_{60}\left(\mathrm{CF}_{3}\right)_{9}(\mathrm{PE}) \mathrm{H}^{-}$and confirmed the addition of a single malonate to the fullerene core, in line with the results of analysis of the ${ }^{1} \mathrm{H}$ and ${ }^{31} \mathrm{P}$ NMR spectra. A Bingel-Hirsch reaction with 60-10-5 and malonate phosphonic ester produced a yellow solution containing two predominant isomers of (60-10-5)(PE) as determined by ${ }^{19} \mathrm{~F}$ NMR (Figure 4). The two isomers were produced in relatively equal amounts according to ${ }^{19} \mathrm{~F}$ and ${ }^{31} \mathrm{P}$ NMR analyses and could not be separated by HPLC using a Buckyprep column. The reaction with 60-10-6 was the least selective of the $\mathrm{C}_{60}\left(\mathrm{CF}_{3}\right)_{10}$ reactions investigated. At least six isomers of (60-10-6)(PE) (see Figure SI-3) were produced that could not be separated by HPLC in $100 \%$ toluene using a Buckyprep column, so the product mixture was not used for further reactions.

The phosphonic ester moieties on one isomer of (60-10-3)(PE) and the mixture of two isomers of (60-10-5)(PE) were then converted to phosphonic acids (PA), $\mathrm{C}_{60}\left(\mathrm{CF}_{3}\right)_{10}(\mathrm{PA})$ (Scheme 1). Phosphonic esters do not bind to $\mathrm{MO}_{x}$ as strongly as phosphonic acids, so the acid derivatives were desired for SAM formation. The phosphonic acid
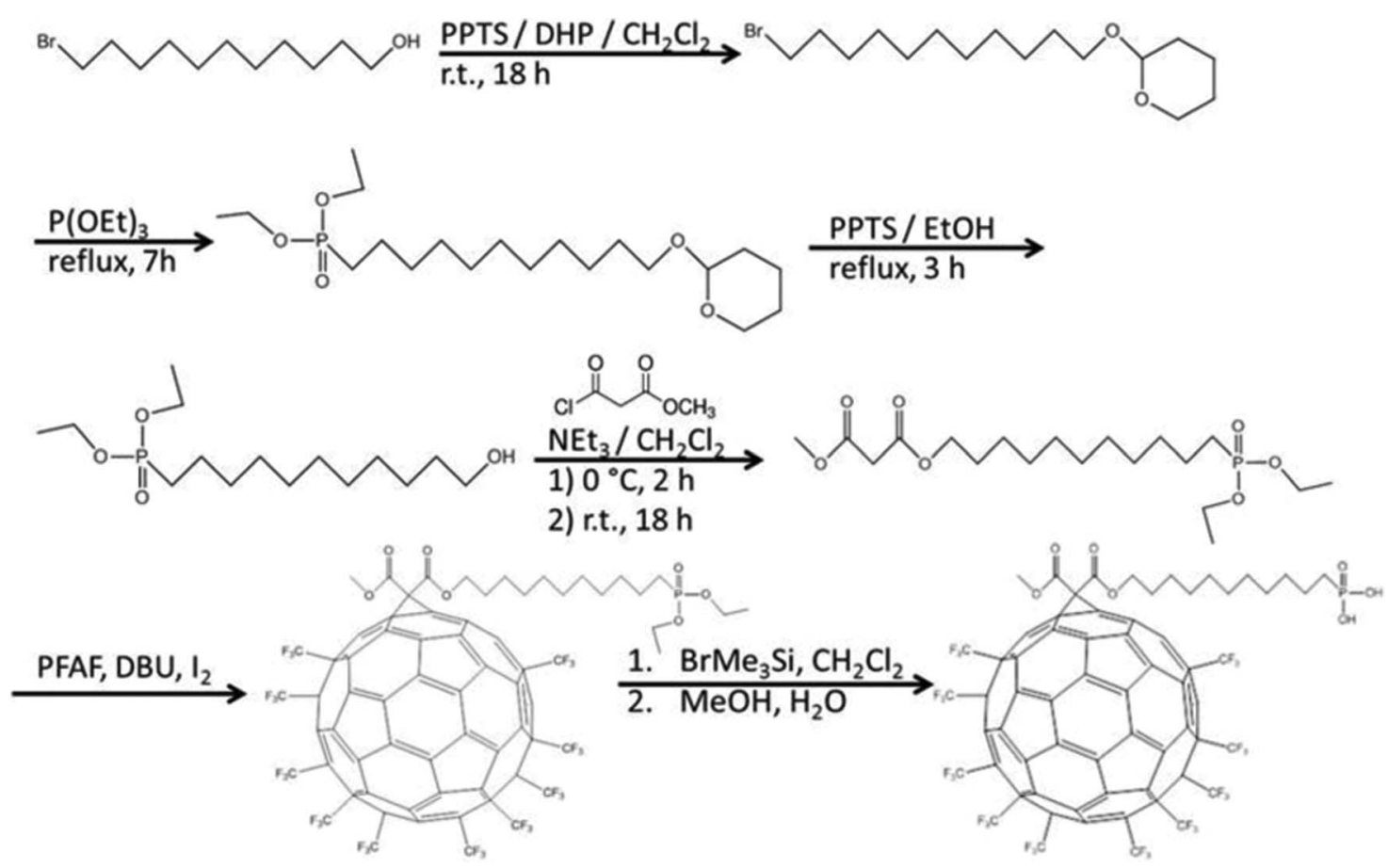

Scheme 1. 


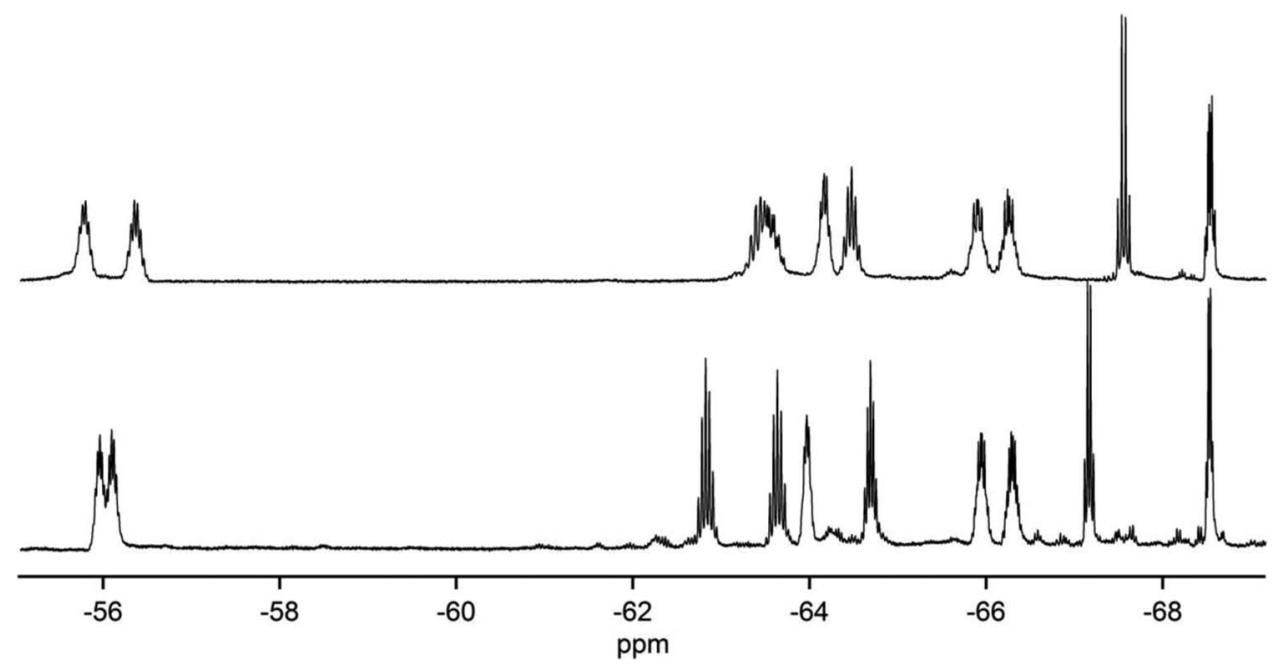

Figure 3. Fluorine-19 NMR spectra of $\mathbf{6 0 - 1 0 - 3}$ (bottom) and HPLC purified (60-10-3)(phosphonic ester) in $\mathrm{CDCl}_{3} . \mathrm{C}_{6} \mathrm{~F}_{6}$ external standard $\delta=-161.4$ ppm.

TMF derivative was synthesized by performing several steps of the reported literature procedure. ${ }^{4-6}$ Each phosphonic ester TMF derivative was dissolved in dry dichloromethane with excess $\mathrm{BrSiMe}_{3}$ for 1 to 3 days before the solvent was removed and $\mathrm{MeOH}$ and $\mathrm{HCl}$ were added. This procedure was repeated several times to maximize acid formation. The solubility of the product mixtures were much lower than the starting phosphonic ester derivatives; this was also observed with other $\mathrm{C}_{60}(\mathrm{PA})$ derivatives. ${ }^{4-6}$ Solubility of the product mixtures decrease in the series of chloroform/methanol, chloroform, toluene, toluene/methanol, DCM, DCM/methanol, methanol, and $\mathrm{CS}_{2}$. A decrease in the ${ }^{1} \mathrm{H}$ NMR signals of the ester moiety relative to other ${ }^{1} \mathrm{H}$ signals indicated a loss of phosphonic ester. Broadening of the $\mathrm{CF}_{3}$ multiplets in ${ }^{19} \mathrm{~F}$ NMR spectra were observed with no shifts in peak positions. Mass spectra (ESI and MALDI in positive and negative mode) showed peaks due to the formation of the phosphonic acid and partially reacted ester products. Complete conversion to the phosphonic acid is not essential, since the partial and full esters will not bind as strongly when forming the SAM and can be washed away to leave a pure monolayer of the $\mathrm{C}_{60}\left(\mathrm{CF}_{3}\right)_{10}$ (phosphonic acid) derivatives.

The successfully synthesized (60-10-3)(PA) sample was then used for preliminary testing its electronic properties in self-assembled monolayer filed-effect transistors (SAMFETs). In brief, SAMs were formed on $\mathrm{AlO}_{\mathrm{x}}$ substrates (formed by atomic layer deposition (ALD) on $\mathrm{Si}$ substrates) by immersion in (60-10-3)(PA) solution. Monolay- ers of (60-10-3)(PA) bound to the $\mathrm{AlO}_{\mathrm{x}}$ surface while weakly bound excess layers and (60-10-3)(PE) were washed away with solvent, which left a single monolayer denoted as (60-10-3)(PA)-SAM. In some cases mixed monolayers were formed starting with $\mathrm{C}_{10} \mathrm{~F}_{17}$ (carboxylic acid) SAMFETs that were exchanged with a solution of (60-10-3)(PA)-SAM according to previous reports. ${ }^{13,14}$

Confirmation of SAM formation was obtained by static contact angle (SCA) and X-ray reflectivity (XRR) measurements. The SCA of (60-10-3)(PA)-SAMs with water were $101.0^{\circ} \pm 0.7^{\circ}$ which is indicative of a hydrophobic surface. In comparison, similarly fabricated non-fluorinated $\mathrm{C}_{60}$-SAMs showed contact angle values $<90^{\circ}$. The vertical electron density profile from XRR shows that the (60-103)(PA) molecules arrange preferentially with the fullerene moieties on top of the SAM (Figure 5). Grazing incidence X-ray diffraction (GID) measurements of (60-10-3)(PA)-SAMs exhibited no diffraction peaks, which indicated that the SAM was (predominantly) amorphous. In comparison, the non-fluorinated $\mathrm{C}_{60}$-SAMs showed a hexagonal crystalline order. ${ }^{15}$

Preliminary testing of SAMFET devices using (60-10-3)(PA)SAMs was carried out. The measured electrical conductivity in FET devices with TMF-based SAMs was below the leakage current through the dielectric and was thus much lower than in non-fluorinated fullerene SAMs. ${ }^{15}$ This decrease in drain current is in accordance with the insulating effects of the fluorous coat on the carbon cage of highly
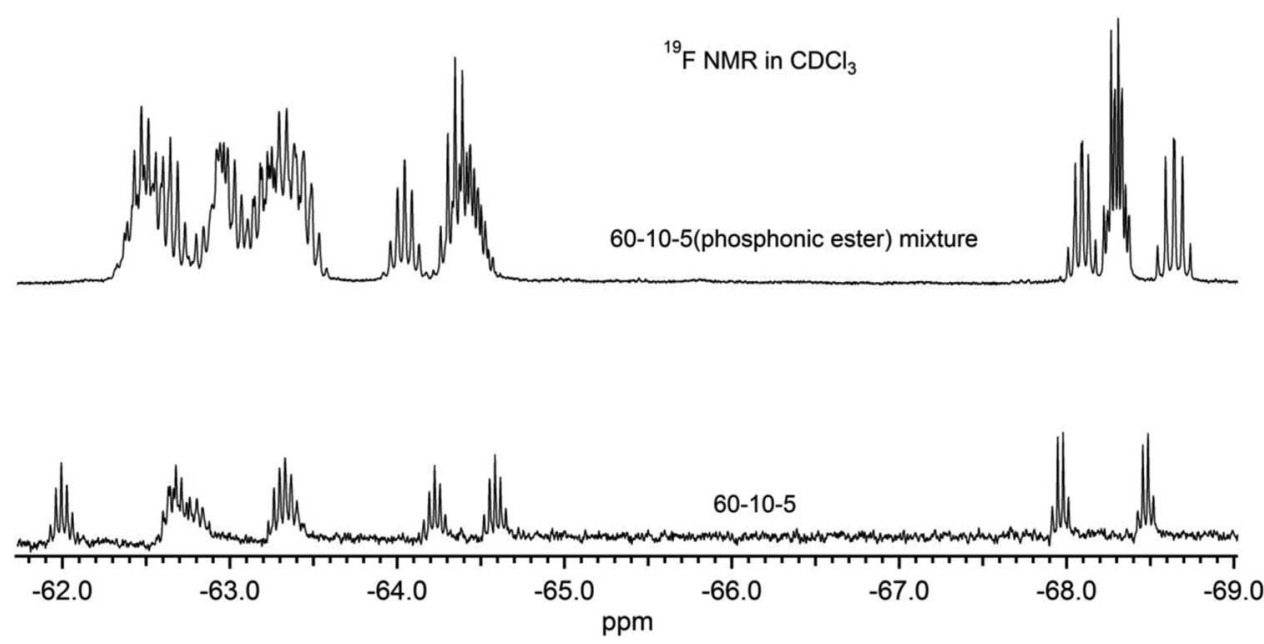

Figure 4. Fluorine-19 NMR spectra of 60-10-5 (bottom) and two isomers of (60-10-5)(phosphonic ester) in $\mathrm{CDCl}_{3} . \mathrm{C}_{6} \mathrm{~F}_{6}$ external standard $\delta=-161.4$ ppm. 


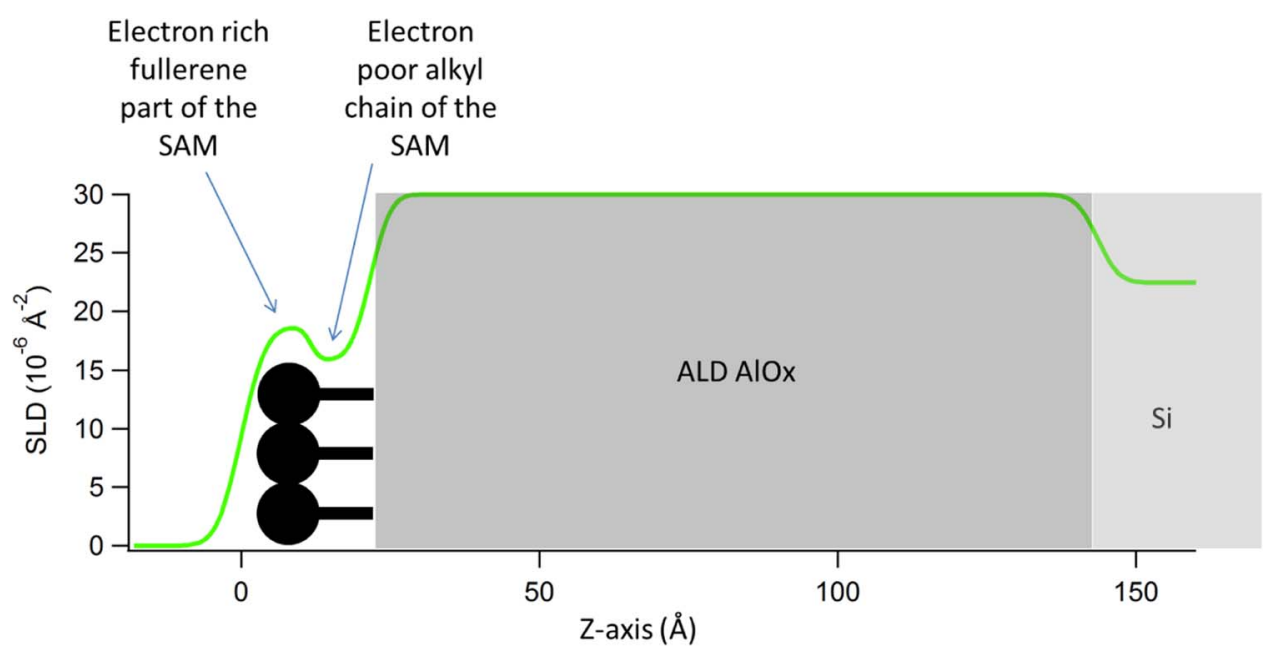

Figure 5. The vertical electron density profile of (60-10-3)(phosphonic acid)-SAMs on ALD $\mathrm{AlO}_{\mathrm{x}}$ plotted over a representative cartoon of the layers.

derivatized TMF. With this respect, fullerene-based electron acceptors with fewer substituents on the core will be more promising candidates for further studies directed toward electronic applications.

\section{Conclusions}

A phosphonic ester malonate was readily added to several $\mathrm{C}_{60}$ TMFs, revealing strong dependence of product distribution on the degree of $\mathrm{CF}_{3}$ substitution on the fullerene core. In general, TMFs with 2 and $4 \mathrm{CF}_{3}$ groups produced multiple adducts which were inseparable under tested conditions. In contrast, two of the three isomers of $\mathrm{C}_{60}\left(\mathrm{CF}_{3}\right)_{10}$ were shown to be fairly selective, producing one and two isomers of monoadducts, respectively, the former with a good isolated yield. Successfully isolated 60-10-3(PE) was converted into a phosphonic acid adduct that was able to strongly bind to $\mathrm{AlO}_{x}$ and form SAMs. The SAMs were more hydrophobic and less crystalline than similar non-fluorinated $\mathrm{C}_{60}$-SAMs. Preliminary FET measurements showed significantly decreased charge transport properties compared to non-fluorinated $\mathrm{C}_{60}-\mathrm{SAMs}$, in accordance with the insulating nature of the fluorous coat on the fullerene molecule.

\section{Acknowledgment}

TTC thanks DAAD for financial support of his research stay in the University of Erlangen, Germany. OVB and SHS are grateful for partial support from the National Science Foundation (grants NSF/CHE1362302, and CHE-1012468). We also acknowledge the European Synchrotron Radiation Facility for provision of synchrotron radiation facilities and we thank Federico Zontone and Oleg Konovalov for assistance in using beamline ID10. TS, AH and MH thank the DFG Collaborative Research Center "Synthetic Carbon Allotropes" (SFB 953).

\section{References}

1. O. V. Boltalina, A. A. Popov, I. V. Kuvychko, N. B. Shustova, and S. H. Strauss, Chem. Rev., 115(2), 1051 (2015).

2. M. Mas-Torrent and C. Rovira, Chem. Rev., 111(8), 4833 (2011).

3. B. Sun and H. Sirringhaus, Nano Letters, 5(12), 2408 (2005).

4. A. Rumpel, M. Novak, J. Walter, B. Braunschweig, M. Halik, and W. Peukert, Langmuir, 27(24), 15016 (2011).

5. M. Burkhardt, A. Jedaa, M. Novak, A. Ebel, K. Voitchovsky, F. Stellacci, A. Hirsch and M. Halik, Adv. Mater, 22(23), 2525 (2010).

6. M. Voigt, M. Klaumunzer, A. Ebel, F. Werner, G. Yang, R. Marczak, E. Spiecker D. M. Guldi, A. Hirsch, and W. Peukert, J. Phys. Chem. C, 115(13), 5561 (2011).

7. N. S. Ovchinnikova, D. V. Ignat'eva, N. B. Tamm, S. M. Avdoshenko, A. A. Goryunkov, I. N. Loffe, V. Y. Markov, S. I. Troyanov, L. N. Sidorov, M. A. Yurovskaya, E. Kemnitz, and L. N. Sidorov, New J. Chem., 32(1), 89 (2008).

8. Y. Takano, M. A. Herranz, I. E. Kareev, S. H. Strauss, O. V. Boltalina, T. Akasaka, and N. Martin, J. Org. Chem., 74(17), 6902 (2009).

9. Y. Takano, M. A. Herranz, N. Martin, G. D. Rojas, D. M. Guldi, I. E. Kareev, S. H. Strauss, O. V. Boltalina, T. Tsuchiya, and T. Akasaka, Chem. Eur. J., 16(18), 5343 (2010)

10. T. T. Clikeman, S. H. M. Deng, S. Avdoshenko, X.-B. Wang, A. A. Popov, S. H. Strauss, and O. V. Boltalina, Chem. Eur. J., 19(45), 15404 (2013).

11. T. T. Clikeman, I. V. Kuvychko, N. B. Shustova, Y.-S. Chen, A. A. Popov, O. V. Boltalina, and S. H. Strauss, Chem. Eur. J., 19(16), 5070 (2013).

12. T. T. Clikeman, S. H. M. Deng, A. A. Popov, X.-B. Wang, S. H. Strauss, and O. V. Boltalina, Phys. Chem. Chem. Phys, 17(1), 551 (2015),

13. T. Lenz, T. Schmaltz, M. Novak, and M. Halik, Langmuir, 28(39), 13900 (2012).

14. Z. Wang, S. Mohammadzadeh, T. Schmaltz, J. Kirschner, A. Khassanov, S. Eigler, U. Mundloch, C. Backes, H.-G. Steinrück, A. Magerl, F. Hauke, A. Hirsch, and M. Halik, ACS Nano, 7(12), 11427 (2013).

15. T. K. Schmaltz, A. Steinruck, H. G. Magerl, A. Hirsch, A. Halik, and M. Nanoscale, 6(21), 13022 (2014) 